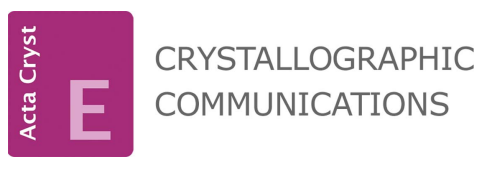

ISSN 2056-9890

Received 15 December 2016

Accepted 8 February 2017

Edited by W. T. A. Harrison, University of Aberdeen, Scotland

Keywords: crystal structure; catalysis; aryl $\mathrm{N}$ oxides; 1-methyl-2H-imidazole 3-N-oxide; hydrate; hydrogen bonding.

CCDC reference: 1531714

Supporting information: this article has supporting information at journals.iucr.org/e

\section{Crystal structure of 1-methylimidazole 3-oxide monohydrate}

\author{
Christopher S. Frampton, ${ }^{a *}$ James I. Murray ${ }^{b}$ and Alan C. Spivey ${ }^{b}$ \\ ${ }^{a}$ Wolfson Centre for Materials Processing, Brunel University London, Kingston Lane, Uxbridge, UB8 3PH, UK, and \\ ${ }^{\mathbf{b}}$ Department of Chemistry, South Kensington Campus, Imperial College London, London, SW7 2AZ, UK. \\ *Correspondence e-mail: chris.frampton@brunel.ac.uk
}

1-Methylimidazole 3- $\mathrm{N}$-oxide (NMI-O) crystallizes as a monohydrate, $\mathrm{C}_{4} \mathrm{H}_{6} \mathrm{~N}_{2} \mathrm{O} \cdot \mathrm{H}_{2} \mathrm{O}$, in the monoclinic space group $P 2_{1}$ with $Z^{\prime}=2$ (molecules $A$ and $B$ ). The imidazole rings display a planar geometry (r.m.s. deviations $=0.0008$ and $0.0002 \AA$ ) and are linked in the crystal structure into infinite zigzag strands of $\cdots$ NMI-O $(A) \cdots \mathrm{OH}_{2} \cdots$ NMI-O $(B) \cdots \mathrm{OH}_{2} \cdots$ units by $\mathrm{O}-\mathrm{H} \cdots \mathrm{O}$ hydrogen bonds. These chains propagate along the $b$-axis direction of the unit cell.

\section{Chemical context}

Aryl- $\mathrm{N}$-oxides are an important class of materials acting as highly efficient catalysts for the phosphorylation of alcohols (Murray et al., 2015) and also for the site-selective phosphoylation of polyols and peptides (Murray et al. 2014). One material in particular, 1-methylimidazole 3- $\mathrm{N}$-oxide, (NMI-O), has been shown to be a highly efficient catalyst for both sulfonylation and silylation procedures (Murray \& Spivey, 2015). Until recently, NMI-O has been somewhat elusive in the literature. The synthesis of NMI-O and its use as a highly efficient catalyst for certain Morita-Baylis-Hillman reactions has been reported (Lin et al., 2005) although no conclusive information on the structural identity of the material synthesized was presented. A recent paper, directed at the synthesis of salts of 1-alkyl-imidazole 3-oxides for use as ionic liquids also reported the synthesis of NMI-O, however all attempts at crystallizing a sample of this material were unsuccessful although two crystalline adducts of NMI-O, a tris (2-thienyl)borane and a silver carbene hexafluoridophosphate, were structurally characterized (Laus et al., 2008). These authors also demonstrated by NMR and subsequent X-ray structural analysis of a related 1,2-dimethylimidazole semiperhydrate material that the likely product reported earlier (Lin et al., 2005) was the 1-methylimidazole semiperhydrate rather than NMI-O itself. We now present a simplified synthesis of MNI-O and the crystal structure of its hydrate.

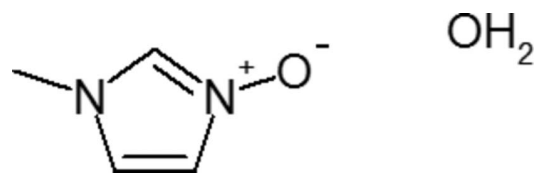

\section{Structural commentary}

The asymmetric unit of the title compound is shown in Fig. 1. It contains two molecules of NMI-O and two fully occupied 


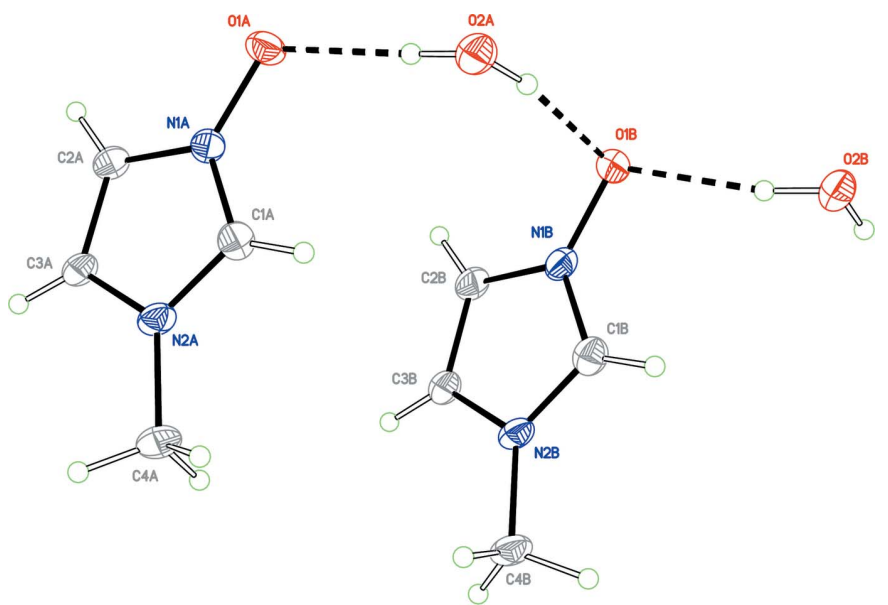

Figure 1

View of the asymmetric unit of the title compound with the atom labelling. Displacement ellipsoids are drawn at the $50 \%$ probability level. The $\mathrm{O}-\mathrm{H} \cdots \mathrm{O}$ hydrogen bonds are shown as dashed lines.

and ordered water molecules, making the overall stoichiometry a monohydrate. A calculated least-squares plane through the five atoms of the imidazole ring $(\mathrm{C} 1, \mathrm{~N} 1, \mathrm{C} 2, \mathrm{C} 3$, N2) for molecules $A$ and $B$ gave r.m.s. deviations from planarity of 0.0008 and $0.0002 \AA$, respectively, with the oxygen atoms of the $\mathrm{N}^{+}-\mathrm{O}^{-}$groups also residing close to the ring plane; O1A, -0.021 (4) $\AA$; O1B, -0.008 (4) $\AA$. The methyl groups lie somewhat farther outside the plane of the ring with displacements of -0.073 (5) $\AA$ for $\mathrm{C} 4 A$ and -0.116 (1) $\AA$ for

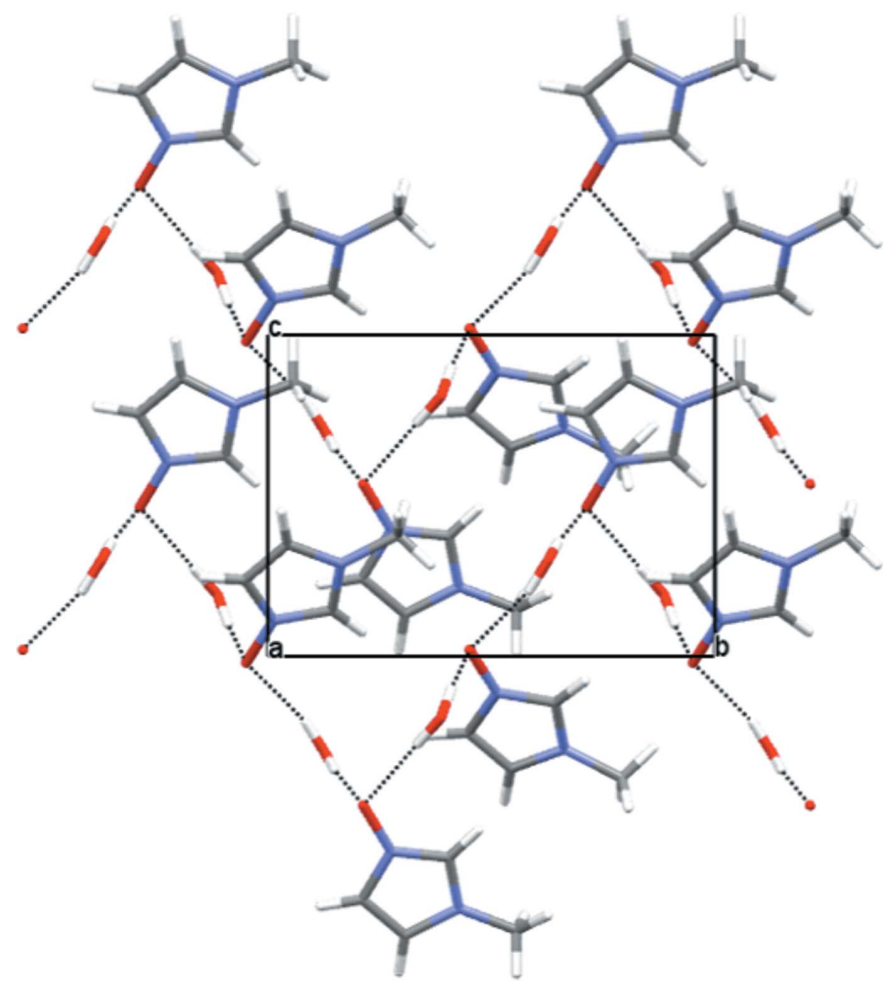

Figure 2

View of the crystal packing down the $a$ axis. The $\mathrm{O}-\mathrm{H} \cdots \mathrm{O}$ hydrogen bonds (see Table 1) are shown as dotted lines.
Table 1

Hydrogen-bond geometry $\left(\AA,^{\circ}\right)$.

\begin{tabular}{|c|c|c|c|c|}
\hline$D-\mathrm{H} \cdots A$ & $D-\mathrm{H}$ & $\mathrm{H} \cdots A$ & $D \cdots A$ & $D-\mathrm{H} \cdots A$ \\
\hline $\mathrm{O} 2 A-\mathrm{H} 2 A A \cdots \mathrm{O} 1 B$ & $1.03(6)$ & $1.73(6)$ & $2.752(3)$ & $172(4)$ \\
\hline $\mathrm{O} 2 A-\mathrm{H} 2 A B \cdots \mathrm{O} 1 A$ & $0.83(5)$ & $1.94(5)$ & $2.773(3)$ & $175(4)$ \\
\hline $\mathrm{O} 2 B-\mathrm{H} 2 B A \cdots \mathrm{O} 1 B$ & $0.83(4)$ & $1.94(4)$ & $2.752(3)$ & $167(4)$ \\
\hline $\mathrm{O} 2 B-\mathrm{H} 2 B B \cdots \mathrm{O} 1 A^{\mathrm{i}}$ & $0.94(5)$ & $1.86(5)$ & $2.790(3)$ & $171(5)$ \\
\hline $\mathrm{C} 1 A-\mathrm{H} 1 A \cdots \mathrm{O} 1 A^{\mathrm{ii}}$ & 0.95 & 2.47 & $3.248(4)$ & 139 \\
\hline $\mathrm{C} 4 A-\mathrm{H} 4 A C \cdots \mathrm{O} 1 A^{\mathrm{ii}}$ & 0.98 & 2.46 & 3.308 (4) & 145 \\
\hline $\mathrm{C} 4 B-\mathrm{H} 4 B C \cdots \mathrm{O} 1 A^{\mathrm{ii}}$ & 0.98 & 2.56 & $3.336(4)$ & 136 \\
\hline $\mathrm{C} 1 B-\mathrm{H} 1 B \cdots \mathrm{O} 1 B^{\mathrm{i}}$ & 0.95 & 2.48 & $3.248(4)$ & 138 \\
\hline $\mathrm{C} 2 B-\mathrm{H} 2 B \cdots \mathrm{O} 2 B^{\mathrm{iii}}$ & 0.95 & 2.41 & $3.298(4)$ & 155 \\
\hline $\mathrm{C} 4 B-\mathrm{H} 4 B A \cdots \mathrm{O} 1 B^{\mathrm{i}}$ & 0.98 & 2.50 & $3.345(4)$ & 144 \\
\hline
\end{tabular}

C4B. The dihedral angle formed between the least-squares planes of the $A$ and $B$ NMI-O molecules is $12.96(16)^{\circ}$. The present data were not of sufficient quality to determine the absolute structure.

\section{Supramolecular features}

In the crystal, the NMI-O and water molecules are linked by $\mathrm{O}-\mathrm{H} \cdots \mathrm{O}$ hydrogen bonds to form an infinite NMI$\mathrm{O} \cdots \mathrm{OH}_{2} \cdots \mathrm{NMI}-\mathrm{O} \cdots \mathrm{OH}_{2} \cdots$ chain propagating along the $b$ axis direction of the unit cell. Each water molecule forms two hydrogen bonds, one to each of the $\mathrm{N}^{+}-\mathrm{O}^{-}$groups of NMI-O molecules $A$ and $B$ with the oxygen atoms of these groups acting as double acceptors from both water molecules (Table 1, Fig. 2). The NMI-O $\cdots \mathrm{OH}_{2} \cdots \mathrm{NMI}-\mathrm{O} \cdots \mathrm{OH}_{2} \cdots$ chains are cross-linked in the crystal structure by weaker $\mathrm{C}-\mathrm{H} \cdots \mathrm{O}$ interactions (Table 1) with $\mathrm{H} \cdots \mathrm{O}$ contacts in the range 2.41$2.56 \AA$.

\section{Database survey}

A search of the Cambridge Structural Database (CSD, Version 5.37 update February 2016; Groom et al., 2016) for the imidazole-3-oxide substructure yielded 16 hits, all of which were genuine examples of substituted imidazole-3-oxides. Closely related examples include 1-hydroxyimidazole-3oxide (DOJKUJ), 1-hydroxy-2-methylimidazole-3-oxide (DOJLAQ), 3-hydroxy-1,2-dimethylimidazolium 1,2-dimethylimidazolium-3-oxide iodide (DOJMUL) and 1,2-dimethylimidazole-3-oxide (DOJNAS) (Laus et al., 2008). For 1-hydroxy-2,4,5-triphenyl-1 $H$-imidazole 3-oxide (JADNAE; Sánchez-Migallón et al. 2003), the $\mathrm{N}^{+}-\mathrm{O}^{-}$bond length was particularly short at 1.276 and $1.278 \AA$ for the two molecules in the asymmetric unit. For the title compound, the $\mathrm{N}^{+}-\mathrm{O}^{-}$ bond lengths are 1.350 (3) and 1.348 (3) $\AA$ for molecules $A$ and $B$, respectively. These values are within the range exhibited for the remaining 15 database entries (1.326-1.368 $)$.

\section{Synthesis and crystallization}

The title compound was synthesized in a three-step, one-pot process in which aqueous glyoxal was condensed with 
Table 2

Experimental details.

\begin{tabular}{|c|c|}
\hline \multicolumn{2}{|l|}{ Crystal data } \\
\hline Chemical formula & $\mathrm{C}_{4} \mathrm{H}_{6} \mathrm{~N}_{2} \mathrm{O} \cdot \mathrm{H}_{2} \mathrm{O}$ \\
\hline$M_{\mathrm{r}}$ & 116.12 \\
\hline Crystal system, space group & Monoclinic, $P 2_{1}$ \\
\hline Temperature (K) & 100 \\
\hline$a, b, c(\AA)$ & 7.5941 (6), 10.0703 (6), 7.8286 (6) \\
\hline$\beta\left({ }^{\circ}\right)$ & $112.402(9)$ \\
\hline$V\left(\AA^{3}\right)$ & $553.51(8)$ \\
\hline$Z$ & 4 \\
\hline Radiation type & $\mathrm{Cu} \mathrm{K \alpha}$ \\
\hline$\mu\left(\mathrm{mm}^{-1}\right)$ & 0.95 \\
\hline Crystal size $(\mathrm{mm})$ & $0.45 \times 0.10 \times 0.05$ \\
\hline \multicolumn{2}{|l|}{ Data collection } \\
\hline Diffractometer & $\begin{array}{l}\text { Rigaku SuperNova, Dualflex, } \\
\text { AtlasS2 }\end{array}$ \\
\hline Absorption correction & $\begin{array}{l}\text { Multi-scan (CrysAlis PRO; Rigaku } \\
\text { OD, 2015) }\end{array}$ \\
\hline$T_{\min }, T_{\max }$ & $0.419,1.000$ \\
\hline $\begin{array}{l}\text { No. of measured, independent and } \\
\text { observed }[I>2 \sigma(I)] \text { reflections }\end{array}$ & $2067,1386,1241$ \\
\hline$R_{\text {int }}$ & 0.023 \\
\hline$(\sin \theta / \lambda)_{\max }\left(\AA^{-1}\right)$ & 0.624 \\
\hline \multicolumn{2}{|l|}{ Refinement } \\
\hline$R\left[F^{2}>2 \sigma\left(F^{2}\right)\right], w R\left(F^{2}\right), S$ & $0.042,0.119,1.01$ \\
\hline No. of reflections & 1386 \\
\hline No. of parameters & 163 \\
\hline No. of restraints & 1 \\
\hline $\mathrm{H}$-atom treatment & $\begin{array}{l}\mathrm{H} \text { atoms treated by a mixture of } \\
\text { independent and constrained } \\
\text { refinement }\end{array}$ \\
\hline$\Delta \rho_{\max }, \Delta \rho_{\min }\left(\mathrm{e} \AA^{-3}\right)$ & $0.21,-0.23$ \\
\hline
\end{tabular}

Computer programs: CrysAlis PRO (Rigaku OD, 2015), SHELXD2014 (Sheldrick et al., 2001), SHELXL2014 (Sheldrick, 2015), SHELXTL (Sheldrick, 2008), Mercury (Macrae et al., 2008) and publCIF (Westrip, 2010).

hydroxylamine hydrochloride in the presence of sodium carbonate to afford the mono-oxime. This intermediate was immediately condensed with methylamine to give the corresponding imine, which cyclo-condenses upon exposure to aqueous formaldehyde to give NMI-O after acidic workup in $\sim 68 \%$ yield (Murray \& Spivey, 2016). The previously reported synthesis also started from glyoxal but required eight steps (Laus et al., 2008). The material was concentrated in vacuo to afford a brown oil, which crystallized overnight as colourless laths in the freezer after exposure to air, forming a monohydrate species. The crystals as prepared were extremely hygroscopic, necessitating a rapid transfer to the cold stream of the diffractometer.

\section{Refinement}

Crystal data, data collection and structure refinement details are summarized in Table 2. The four water $\mathrm{H}$ atoms were located in a Fourier difference map and freely refined. All the remaining $\mathrm{H}$ atoms were placed geometrically in idealized positions and allowed to ride on their parent atoms: $\mathrm{C}-\mathrm{H}=$ $0.95-0.98 \AA$ with $U_{\text {iso }}(\mathrm{H})=1.5 U_{\text {eq }}(\mathrm{C}$-methyl $)$ and $U_{\text {iso }}(\mathrm{H})=$ $1.2 U_{\text {eq }}(\mathrm{C})$ for other $\mathrm{H}$ atoms. The data were not of a sufficient quality to reliably determine the absolute structure.

\section{References}

Groom, C. R., Bruno, I. J., Lightfoot, M. P. \& Ward, S. C. (2016). Acta Cryst. B72, 171-179.

Laus, G., Schwärtzler, A., Bentivoglioa, G., Hummel, M., Kahlenberg, V., Wurst, K., Kristeva, E., Schütz, J., Kopacka, H., Kreutz, C., Bonn, G., Andriyko, Y., Nauer, G. \& Schottenberger, H. (2008). Z. Naturforsch. Teil B, 63, 447-464.

Lin, Y.-S., Liu, C.-W. \& Tsai, T. Y. R. (2005). Tetrahedron Lett. 46, 1859-1861.

Macrae, C. F., Bruno, I. J., Chisholm, J. A., Edgington, P. R., McCabe, P., Pidcock, E., Rodriguez-Monge, L., Taylor, R., van de Streek, J. \& Wood, P. A. (2008). J. Appl. Cryst. 41, 466-470.

Murray, J. I. \& Spivey, A. C. (2016). Org. Synth. 93, 331-340.

Murray, J. I. \& Spivey, A. C. (2015). Adv. Synth. Catal. 357, 38253830.

Murray, J. I., Woscholski, R. \& Spivey, A. C. (2014). Chem. Commun. 50, 13608-13611.

Murray, J. I., Woscholski, R. \& Spivey, A. C. (2015). Synlett, 26, $985-$ 990.

Rigaku OD (2015). CrysAlis PRO. Rigaku Oxford Diffraction, Oxford, UK.

Sánchez-Migallón, A., de la Hoz, A., López, C., Claramunt, R. M., Infantes, L., Motherwell, S., Shankland, K., Nowell, H., Alkorta, I. \& Elguero, J. (2003). Helv. Chim. Acta, 86, 1026-1039.

Sheldrick, G. M. (2008). Acta Cryst. A64, 112-122.

Sheldrick, G. M. (2015). Acta Cryst. C71, 3-8.

Sheldrick, G. M., Hauptman, H. A., Weeks, C. M., Miller, M. \& Usón, I. (2001). International Tables for Crystallography, Vol. F, edited by E. Arnold \& M. Rossmann, pp. 333-351. Dordrecht: Kluwer.

Westrip, S. P. (2010). J. Appl. Cryst. 43, 920-925. 


\section{supporting information}

Acta Cryst. (2017). E73, 372-374 [https://doi.org/10.1107/S2056989017002079]

\section{Crystal structure of 1-methylimidazole 3-oxide monohydrate}

\section{Christopher S. Frampton, James I. Murray and Alan C. Spivey}

Computing details

Data collection: CrysAlis PRO (Rigaku OD, 2015); cell refinement: CrysAlis PRO (Rigaku OD, 2015); data reduction: CrysAlis PRO (Rigaku OD, 2015); program(s) used to solve structure: SHELXD2014 (Sheldrick et al., 2001); program(s) used to refine structure: SHELXL2014 (Sheldrick, 2015b); molecular graphics: SHELXTL (Sheldrick, 2008) and Mercury (Macrae et al., 2008); software used to prepare material for publication: SHELXTL (Sheldrick, 2008), Mercury (Macrae et al., 2008) and publCIF (Westrip, 2010).

1-Methylimidazole 3-N-oxide monohydrate

Crystal data

\section{$\mathrm{C}_{4} \mathrm{H}_{6} \mathrm{~N}_{2} \mathrm{O} \cdot \mathrm{H}_{2} \mathrm{O}$}

$M_{r}=116.12$

Monoclinic, $P 2$

$a=7.5941(6) \AA$

$b=10.0703(6) \AA$

$c=7.8286(6) \AA$

$\beta=112.402(9)^{\circ}$

$V=553.51(8) \AA^{3}$

$Z=4$

\section{Data collection}

Rigaku SuperNova, Dualflex, AtlasS2 diffractometer

Radiation source: fine-focus sealed X-ray tube, Enhance (Cu) X-ray Source

Detector resolution: 5.2921 pixels $\mathrm{mm}^{-1}$ $\omega$ scans

Absorption correction: multi-scan

(CrysAlis PRO; Rigaku OD, 2015)

$T_{\min }=0.419, T_{\max }=1.000$

Refinement

Refinement on $F^{2}$

Least-squares matrix: full

$R\left[F^{2}>2 \sigma\left(F^{2}\right)\right]=0.042$

$w R\left(F^{2}\right)=0.119$

$S=1.01$

1386 reflections

163 parameters

1 restraint
$F(000)=248$

$D_{\mathrm{x}}=1.393 \mathrm{Mg} \mathrm{m}^{-3}$

$\mathrm{Cu} K \alpha$ radiation, $\lambda=1.54184 \AA$

Cell parameters from 1007 reflections

$\theta=6.3-74.8^{\circ}$

$\mu=0.95 \mathrm{~mm}^{-1}$

$T=100 \mathrm{~K}$

Lath, colourless

$0.45 \times 0.10 \times 0.05 \mathrm{~mm}$

2067 measured reflections

1386 independent reflections

1241 reflections with $I>2 \sigma(I)$

$R_{\text {int }}=0.023$

$\theta_{\max }=74.3^{\circ}, \theta_{\min }=6.1^{\circ}$

$h=-9 \rightarrow 8$

$k=-12 \rightarrow 5$

$l=-9 \rightarrow 7$

Primary atom site location: structure-invariant direct methods

Hydrogen site location: mixed

$\mathrm{H}$ atoms treated by a mixture of independent and constrained refinement

$w=1 /\left[\sigma^{2}\left(F_{\mathrm{o}}^{2}\right)+(0.075 P)^{2}\right]$

where $P=\left(F_{\mathrm{o}}^{2}+2 F_{\mathrm{c}}{ }^{2}\right) / 3$

$(\Delta / \sigma)_{\max }<0.001$

$\Delta \rho_{\max }=0.21 \mathrm{e} \AA^{-3}$

$\Delta \rho_{\min }=-0.23$ e $\AA^{-3}$ 


\section{Special details}

Geometry. All esds (except the esd in the dihedral angle between two 1.s. planes) are estimated using the full covariance matrix. The cell esds are taken into account individually in the estimation of esds in distances, angles and torsion angles; correlations between esds in cell parameters are only used when they are defined by crystal symmetry. An approximate (isotropic) treatment of cell esds is used for estimating esds involving l.s. planes.

Fractional atomic coordinates and isotropic or equivalent isotropic displacement parameters $\left(\AA^{2}\right)$

\begin{tabular}{|c|c|c|c|c|}
\hline & $x$ & $y$ & $z$ & $U_{\text {iso }} * / U_{\text {eq }}$ \\
\hline O1A & $0.5193(3)$ & $0.2133(2)$ & $0.5360(3)$ & $0.0218(5)$ \\
\hline N1A & $0.3842(3)$ & 0.2757 (3) & $0.3934(3)$ & $0.0176(6)$ \\
\hline $\mathrm{N} 2 \mathrm{~A}$ & $0.2057(4)$ & $0.4269(3)$ & $0.2161(4)$ & $0.0189(6)$ \\
\hline $\mathrm{C} 1 \mathrm{~A}$ & $0.3553(4)$ & $0.4046(3)$ & $0.3747(4)$ & $0.0193(6)$ \\
\hline $\mathrm{H} 1 \mathrm{~A}$ & 0.4276 & 0.4707 & 0.4589 & $0.023^{*}$ \\
\hline $\mathrm{C} 2 \mathrm{~A}$ & $0.2517(4)$ & 0.2107 (3) & $0.2439(4)$ & $0.0185(6)$ \\
\hline $\mathrm{H} 2 \mathrm{~A}$ & 0.2413 & 0.1176 & 0.2231 & $0.022 *$ \\
\hline $\mathrm{C} 3 \mathrm{~A}$ & $0.1392(4)$ & $0.3066(3)$ & $0.1325(4)$ & $0.0185(6)$ \\
\hline H3A & 0.0348 & 0.2931 & 0.0187 & $0.022 *$ \\
\hline $\mathrm{C} 4 \mathrm{~A}$ & $0.1200(4)$ & $0.5563(3)$ & $0.1484(5)$ & $0.0231(7)$ \\
\hline H4AA & 0.0184 & 0.5739 & 0.1936 & $0.035^{*}$ \\
\hline $\mathrm{H} 4 \mathrm{AB}$ & 0.0667 & 0.5562 & 0.0130 & $0.035^{*}$ \\
\hline $\mathrm{H} 4 \mathrm{AC}$ & 0.2176 & 0.6256 & 0.1934 & $0.035^{*}$ \\
\hline $\mathrm{O} 2 \mathrm{~A}$ & $0.7412(3)$ & $0.3868(2)$ & $0.8081(3)$ & $0.0237(5)$ \\
\hline H2AA & $0.675(7)$ & $0.410(6)$ & $0.898(7)$ & $0.063(16)^{*}$ \\
\hline $\mathrm{H} 2 \mathrm{AB}$ & $0.669(6)$ & $0.335(5)$ & $0.729(6)$ & $0.039(13)^{*}$ \\
\hline O1B & $0.5360(3)$ & $0.4513(2)$ & $1.0200(3)$ & $0.0226(5)$ \\
\hline N1B & 0.3895 (4) & $0.5126(3)$ & $0.8873(3)$ & $0.0188(6)$ \\
\hline $\mathrm{N} 2 \mathrm{~B}$ & $0.2080(4)$ & $0.6632(3)$ & $0.7115(4)$ & $0.0183(6)$ \\
\hline $\mathrm{C} 1 \mathrm{~B}$ & $0.3733(4)$ & $0.6430(3)$ & $0.8560(4)$ & $0.0203(7)$ \\
\hline H1B & 0.4617 & 0.7092 & 0.9231 & $0.024^{*}$ \\
\hline $\mathrm{C} 2 \mathrm{~B}$ & $0.2336(4)$ & 0.4478 (3) & $0.7620(4)$ & $0.0201(6)$ \\
\hline $\mathrm{H} 2 \mathrm{~B}$ & 0.2105 & 0.3548 & 0.7544 & $0.024^{*}$ \\
\hline C3B & $0.1191(4)$ & $0.5435(3)$ & 0.6509 (4) & $0.0194(6)$ \\
\hline H3B & 0.0007 & 0.5300 & 0.5510 & $0.023^{*}$ \\
\hline C4B & $0.1431(4)$ & 0.7915 (3) & $0.6217(5)$ & $0.0222(7)$ \\
\hline H4BA & 0.1842 & 0.8624 & 0.7144 & $0.033^{*}$ \\
\hline H4BB & 0.0038 & 0.7916 & 0.5622 & $0.033^{*}$ \\
\hline H4BC & 0.1978 & 0.8065 & 0.5284 & $0.033^{*}$ \\
\hline $\mathrm{O} 2 \mathrm{~B}$ & $0.7272(3)$ & $0.6268(2)$ & $1.2987(3)$ & $0.0242(6)$ \\
\hline $\mathrm{H} 2 \mathrm{BA}$ & $0.663(6)$ & $0.585(5)$ & $1.205(5)$ & $0.023(10)^{*}$ \\
\hline $\mathrm{H} 2 \mathrm{BB}$ & $0.638(7)$ & $0.648(6)$ & $1.351(7)$ & $0.057(15)^{*}$ \\
\hline
\end{tabular}

Atomic displacement parameters $\left(\AA^{2}\right)$

\begin{tabular}{lllllll}
\hline & $U^{11}$ & $U^{22}$ & $U^{33}$ & $U^{12}$ & $U^{13}$ & $U^{23}$ \\
\hline O1A & $0.0226(10)$ & $0.0189(12)$ & $0.0202(12)$ & $0.0033(9)$ & $0.0039(10)$ & $0.0048(10)$ \\
N1A & $0.0212(12)$ & $0.0148(13)$ & $0.0171(12)$ & $0.0005(9)$ & $0.0075(10)$ & $0.0015(10)$ \\
N2A & $0.0236(12)$ & $0.0108(14)$ & $0.0229(13)$ & $0.0007(10)$ & $0.0095(11)$ & $0.0008(10)$
\end{tabular}




$\begin{array}{lllllll}\text { C1A } & 0.0210(13) & 0.0169(16) & 0.0196(15) & -0.0029(12) & 0.0071(12) & -0.0001(11) \\ \text { C2A } & 0.0228(14) & 0.0103(15) & 0.0223(15) & -0.0013(12) & 0.0084(13) & -0.0007(12) \\ \text { C3A } & 0.0227(14) & 0.0101(14) & 0.0219(15) & -0.0012(11) & 0.0077(12) & -0.0025(12) \\ \text { C4A } & 0.0290(15) & 0.0107(15) & 0.0300(16) & 0.0013(12) & 0.0117(14) & 0.0027(13) \\ \text { O2A } & 0.0248(11) & 0.0177(13) & 0.0263(12) & -0.0010(9) & 0.0071(10) & -0.0023(10) \\ \text { O1B } & 0.0256(11) & 0.0192(11) & 0.0185(10) & 0.0039(9) & 0.0032(9) & -0.0010(9) \\ \text { N1B } & 0.0243(13) & 0.0137(14) & 0.0187(13) & 0.0015(10) & 0.0086(11) & -0.0017(9) \\ \text { N2B } & 0.0229(12) & 0.0110(13) & 0.0220(12) & 0.0005(10) & 0.0097(10) & -0.0001(10) \\ \text { C1B } & 0.0230(15) & 0.0181(16) & 0.0209(14) & -0.0025(12) & 0.0097(13) & -0.0020(12) \\ \text { C2B } & 0.0263(15) & 0.0111(14) & 0.0224(14) & -0.0015(12) & 0.0087(12) & -0.0012(11) \\ \text { C3B } & 0.0217(13) & 0.0139(15) & 0.0210(13) & -0.0024(12) & 0.0063(12) & -0.0032(12) \\ \text { C4B } & 0.0280(15) & 0.0108(15) & 0.0279(16) & 0.0015(13) & 0.0107(14) & 0.0025(12) \\ \text { O2B } & 0.0265(12) & 0.0200(14) & 0.0251(11) & -0.0013(10) & 0.0086(10) & -0.0053(10) \\ & & & & & \end{array}$

Geometric parameters $\left(\AA,{ }^{\circ}\right)$

\begin{tabular}{|c|c|c|c|}
\hline $\mathrm{O} 1 \mathrm{~A}-\mathrm{N} 1 \mathrm{~A}$ & $1.350(3)$ & $\mathrm{O} 1 \mathrm{~B}-\mathrm{N} 1 \mathrm{~B}$ & $1.348(3)$ \\
\hline $\mathrm{N} 1 \mathrm{~A}-\mathrm{C} 1 \mathrm{~A}$ & $1.315(4)$ & $\mathrm{N} 1 \mathrm{~B}-\mathrm{C} 1 \mathrm{~B}$ & $1.332(4)$ \\
\hline $\mathrm{N} 1 \mathrm{~A}-\mathrm{C} 2 \mathrm{~A}$ & $1.384(4)$ & $\mathrm{N} 1 \mathrm{~B}-\mathrm{C} 2 \mathrm{~B}$ & $1.380(4)$ \\
\hline $\mathrm{N} 2 \mathrm{~A}-\mathrm{C} 1 \mathrm{~A}$ & $1.344(4)$ & $\mathrm{N} 2 \mathrm{~B}-\mathrm{C} 1 \mathrm{~B}$ & $1.348(4)$ \\
\hline $\mathrm{N} 2 \mathrm{~A}-\mathrm{C} 3 \mathrm{~A}$ & $1.378(4)$ & $\mathrm{N} 2 \mathrm{~B}-\mathrm{C} 3 \mathrm{~B}$ & $1.374(4)$ \\
\hline $\mathrm{N} 2 \mathrm{~A}-\mathrm{C} 4 \mathrm{~A}$ & $1.463(4)$ & $\mathrm{N} 2 \mathrm{~B}-\mathrm{C} 4 \mathrm{~B}$ & $1.464(4)$ \\
\hline $\mathrm{C} 1 \mathrm{~A}-\mathrm{H} 1 \mathrm{~A}$ & 0.9500 & $\mathrm{C} 1 \mathrm{~B}-\mathrm{H} 1 \mathrm{~B}$ & 0.9500 \\
\hline $\mathrm{C} 2 \mathrm{~A}-\mathrm{C} 3 \mathrm{~A}$ & $1.362(4)$ & $\mathrm{C} 2 \mathrm{~B}-\mathrm{C} 3 \mathrm{~B}$ & $1.366(4)$ \\
\hline $\mathrm{C} 2 \mathrm{~A}-\mathrm{H} 2 \mathrm{~A}$ & 0.9500 & $\mathrm{C} 2 \mathrm{~B}-\mathrm{H} 2 \mathrm{~B}$ & 0.9500 \\
\hline $\mathrm{C} 3 \mathrm{~A}-\mathrm{H} 3 \mathrm{~A}$ & 0.9500 & $\mathrm{C} 3 \mathrm{~B}-\mathrm{H} 3 \mathrm{~B}$ & 0.9500 \\
\hline $\mathrm{C} 4 \mathrm{~A}-\mathrm{H} 4 \mathrm{AA}$ & 0.9800 & $\mathrm{C} 4 \mathrm{~B}-\mathrm{H} 4 \mathrm{BA}$ & 0.9800 \\
\hline $\mathrm{C} 4 \mathrm{~A}-\mathrm{H} 4 \mathrm{AB}$ & 0.9800 & $\mathrm{C} 4 \mathrm{~B}-\mathrm{H} 4 \mathrm{BB}$ & 0.9800 \\
\hline $\mathrm{C} 4 \mathrm{~A}-\mathrm{H} 4 \mathrm{AC}$ & 0.9800 & $\mathrm{C} 4 \mathrm{~B}-\mathrm{H} 4 \mathrm{BC}$ & 0.9800 \\
\hline $\mathrm{O} 2 \mathrm{~A}-\mathrm{H} 2 \mathrm{AA}$ & $1.03(6)$ & $\mathrm{O} 2 \mathrm{~B}-\mathrm{H} 2 \mathrm{BA}$ & $0.83(4)$ \\
\hline $\mathrm{O} 2 \mathrm{~A}-\mathrm{H} 2 \mathrm{AB}$ & $0.83(5)$ & $\mathrm{O} 2 \mathrm{~B}-\mathrm{H} 2 \mathrm{BB}$ & $0.94(5)$ \\
\hline $\mathrm{C} 1 \mathrm{~A}-\mathrm{N} 1 \mathrm{~A}-\mathrm{O} 1 \mathrm{~A}$ & $126.5(3)$ & $\mathrm{C} 1 \mathrm{~B}-\mathrm{N} 1 \mathrm{~B}-\mathrm{O} 1 \mathrm{~B}$ & $125.7(3)$ \\
\hline $\mathrm{C} 1 \mathrm{~A}-\mathrm{N} 1 \mathrm{~A}-\mathrm{C} 2 \mathrm{~A}$ & $109.6(3)$ & $\mathrm{C} 1 \mathrm{~B}-\mathrm{N} 1 \mathrm{~B}-\mathrm{C} 2 \mathrm{~B}$ & $110.0(3)$ \\
\hline $\mathrm{O} 1 \mathrm{~A}-\mathrm{N} 1 \mathrm{~A}-\mathrm{C} 2 \mathrm{~A}$ & $123.9(3)$ & $\mathrm{O} 1 \mathrm{~B}-\mathrm{N} 1 \mathrm{~B}-\mathrm{C} 2 \mathrm{~B}$ & $124.3(3)$ \\
\hline $\mathrm{C} 1 \mathrm{~A}-\mathrm{N} 2 \mathrm{~A}-\mathrm{C} 3 \mathrm{~A}$ & $108.6(3)$ & $\mathrm{C} 1 \mathrm{~B}-\mathrm{N} 2 \mathrm{~B}-\mathrm{C} 3 \mathrm{~B}$ & $109.6(3)$ \\
\hline $\mathrm{C} 1 \mathrm{~A}-\mathrm{N} 2 \mathrm{~A}-\mathrm{C} 4 \mathrm{~A}$ & $125.8(3)$ & $\mathrm{C} 1 \mathrm{~B}-\mathrm{N} 2 \mathrm{~B}-\mathrm{C} 4 \mathrm{~B}$ & $124.9(3)$ \\
\hline $\mathrm{C} 3 \mathrm{~A}-\mathrm{N} 2 \mathrm{~A}-\mathrm{C} 4 \mathrm{~A}$ & $125.4(3)$ & $\mathrm{C} 3 \mathrm{~B}-\mathrm{N} 2 \mathrm{~B}-\mathrm{C} 4 \mathrm{~B}$ & $125.3(3)$ \\
\hline $\mathrm{N} 1 \mathrm{~A}-\mathrm{C} 1 \mathrm{~A}-\mathrm{N} 2 \mathrm{~A}$ & $108.3(3)$ & $\mathrm{N} 1 \mathrm{~B}-\mathrm{C} 1 \mathrm{~B}-\mathrm{N} 2 \mathrm{~B}$ & $107.1(3)$ \\
\hline $\mathrm{N} 1 \mathrm{~A}-\mathrm{C} 1 \mathrm{~A}-\mathrm{H} 1 \mathrm{~A}$ & 125.8 & $\mathrm{~N} 1 \mathrm{~B}-\mathrm{C} 1 \mathrm{~B}-\mathrm{H} 1 \mathrm{~B}$ & 126.4 \\
\hline $\mathrm{N} 2 \mathrm{~A}-\mathrm{C} 1 \mathrm{~A}-\mathrm{H} 1 \mathrm{~A}$ & 125.8 & $\mathrm{~N} 2 \mathrm{~B}-\mathrm{C} 1 \mathrm{~B}-\mathrm{H} 1 \mathrm{~B}$ & 126.4 \\
\hline $\mathrm{C} 3 \mathrm{~A}-\mathrm{C} 2 \mathrm{~A}-\mathrm{N} 1 \mathrm{~A}$ & $106.4(3)$ & $\mathrm{C} 3 \mathrm{~B}-\mathrm{C} 2 \mathrm{~B}-\mathrm{N} 1 \mathrm{~B}$ & $106.5(3)$ \\
\hline $\mathrm{C} 3 \mathrm{~A}-\mathrm{C} 2 \mathrm{~A}-\mathrm{H} 2 \mathrm{~A}$ & 126.8 & $\mathrm{C} 3 \mathrm{~B}-\mathrm{C} 2 \mathrm{~B}-\mathrm{H} 2 \mathrm{~B}$ & 126.7 \\
\hline $\mathrm{N} 1 \mathrm{~A}-\mathrm{C} 2 \mathrm{~A}-\mathrm{H} 2 \mathrm{~A}$ & 126.8 & $\mathrm{~N} 1 \mathrm{~B}-\mathrm{C} 2 \mathrm{~B}-\mathrm{H} 2 \mathrm{~B}$ & 126.7 \\
\hline $\mathrm{C} 2 \mathrm{~A}-\mathrm{C} 3 \mathrm{~A}-\mathrm{N} 2 \mathrm{~A}$ & $107.0(3)$ & $\mathrm{C} 2 \mathrm{~B}-\mathrm{C} 3 \mathrm{~B}-\mathrm{N} 2 \mathrm{~B}$ & $106.8(3)$ \\
\hline $\mathrm{C} 2 \mathrm{~A}-\mathrm{C} 3 \mathrm{~A}-\mathrm{H} 3 \mathrm{~A}$ & 126.5 & $\mathrm{C} 2 \mathrm{~B}-\mathrm{C} 3 \mathrm{~B}-\mathrm{H} 3 \mathrm{~B}$ & 126.6 \\
\hline $\mathrm{N} 2 \mathrm{~A}-\mathrm{C} 3 \mathrm{~A}-\mathrm{H} 3 \mathrm{~A}$ & 126.5 & $\mathrm{~N} 2 \mathrm{~B}-\mathrm{C} 3 \mathrm{~B}-\mathrm{H} 3 \mathrm{~B}$ & 126.6 \\
\hline $\mathrm{N} 2 \mathrm{~A}-\mathrm{C} 4 \mathrm{~A}-\mathrm{H} 4 \mathrm{AA}$ & 109.5 & $\mathrm{~N} 2 \mathrm{~B}-\mathrm{C} 4 \mathrm{~B}-\mathrm{H} 4 \mathrm{BA}$ & 109.5 \\
\hline
\end{tabular}




$\begin{array}{ll}\mathrm{N} 2 \mathrm{~A}-\mathrm{C} 4 \mathrm{~A}-\mathrm{H} 4 \mathrm{AB} & 109.5 \\ \mathrm{H} 4 \mathrm{AA}-\mathrm{C} 4 \mathrm{~A}-\mathrm{H} 4 \mathrm{AB} & 109.5 \\ \mathrm{~N} 2 \mathrm{~A}-\mathrm{C} 4 \mathrm{~A}-\mathrm{H} 4 \mathrm{AC} & 109.5 \\ \mathrm{H} 4 \mathrm{AA}-\mathrm{C} 4 \mathrm{~A}-\mathrm{H} 4 \mathrm{AC} & 109.5 \\ \mathrm{H} 4 \mathrm{AB}-\mathrm{C} 4 \mathrm{~A}-\mathrm{H} 4 \mathrm{AC} & 109.5 \\ \mathrm{H} 2 \mathrm{AA}-\mathrm{O} 2 \mathrm{~A}-\mathrm{H} 2 \mathrm{AB} & 107(4) \\ & \\ \mathrm{O} 1 \mathrm{~A}-\mathrm{N} 1 \mathrm{~A}-\mathrm{C} 1 \mathrm{~A}-\mathrm{N} 2 \mathrm{~A} & -178.9(2) \\ \mathrm{C} 2 \mathrm{~A}-\mathrm{N} 1 \mathrm{~A}-\mathrm{C} 1 \mathrm{~A}-\mathrm{N} 2 \mathrm{~A} & 0.2(3) \\ \mathrm{C} 3 \mathrm{~A}-\mathrm{N} 2 \mathrm{~A}-\mathrm{C} 1 \mathrm{~A}-\mathrm{N} 1 \mathrm{~A} & -0.1(3) \\ \mathrm{C} 4 \mathrm{~A}-\mathrm{N} 2 \mathrm{~A}-\mathrm{C} 1 \mathrm{~A}-\mathrm{N} 1 \mathrm{~A} & 176.4(3) \\ \mathrm{C} 1 \mathrm{~A}-\mathrm{N} 1 \mathrm{~A}-\mathrm{C} 2 \mathrm{~A}-\mathrm{C} 3 \mathrm{~A} & -0.2(3) \\ \mathrm{O} 1 \mathrm{~A}-\mathrm{N} 1 \mathrm{~A}-\mathrm{C} 2 \mathrm{~A}-\mathrm{C} 3 \mathrm{~A} & 179.0(2) \\ \mathrm{N} 1 \mathrm{~A}-\mathrm{C} 2 \mathrm{~A}-\mathrm{C} 3 \mathrm{~A}-\mathrm{N} 2 \mathrm{~A} & 0.1(3) \\ \mathrm{C} 1 \mathrm{~A}-\mathrm{N} 2 \mathrm{~A}-\mathrm{C} 3 \mathrm{~A}-\mathrm{C} 2 \mathrm{~A} & 0.0(3) \\ \mathrm{C} 4 \mathrm{~A}-\mathrm{N} 2 \mathrm{~A}-\mathrm{C} 3 \mathrm{~A}-\mathrm{C} 2 \mathrm{~A} & -176.6(3)\end{array}$

N2B-C4B-H4BB
H4BA-C4B-H4BB
N2B-C4B-H4BC
H4BA-C4B-H4BC
H4BB-C4B-H4BC
H2BA-O2B-H2BB
O1B-N1B-C1B-N2B
C2B-N1B-C1B-N2B
C3B-N2B-C1B-N1B
C4B-N2B-C1B-N1B
C1B-N1B-C2B-C3B
O1B-N1B-C2B-C3B
N1B-C2B-C3B-N2B
C1B-N2B-C3B-C2B
C4B-N2B-C3B-C2B

109.5

109.5

109.5

109.5

109.5

$103(4)$

$179.6(2)$

$0.0(4)$

$0.0(3)$

$-174.4(3)$

$0.0(4)$

$-179.6(2)$

$0.0(3)$

$0.0(3)$

$174.4(3)$

Hydrogen-bond geometry $\left(A,{ }^{\circ}\right)$

\begin{tabular}{lllll}
\hline$D-\mathrm{H} \cdots A$ & $D-\mathrm{H}$ & $\mathrm{H} \cdots A$ & $D \cdots A$ & $D-\mathrm{H} \cdots A$ \\
\hline $\mathrm{O} 2 A-\mathrm{H} 2 A A \cdots \mathrm{O} 1 B$ & $1.03(6)$ & $1.73(6)$ & $2.752(3)$ & $172(4)$ \\
$\mathrm{O} 2 A-\mathrm{H} 2 A B \cdots \mathrm{O} 1 A$ & $0.83(5)$ & $1.94(5)$ & $2.773(3)$ & $175(4)$ \\
$\mathrm{O} 2 B-\mathrm{H} 2 B A \cdots \mathrm{O} 1 B$ & $0.83(4)$ & $1.94(4)$ & $2.752(3)$ & $167(4)$ \\
$\mathrm{O} 2 B-\mathrm{H} 2 B B \cdots \mathrm{O} 1 A^{\mathrm{i}}$ & $0.94(5)$ & $1.86(5)$ & $2.790(3)$ & $171(5)$ \\
$\mathrm{C} 1 A-\mathrm{H} 1 A \cdots \mathrm{O} 1 A^{i i}$ & 0.95 & 2.47 & $3.248(4)$ & 139 \\
$\mathrm{C} 4 A-\mathrm{H} 4 A C \cdots \mathrm{O} 1 A^{\mathrm{ii}}$ & 0.98 & 2.46 & $3.308(4)$ & 145 \\
$\mathrm{C} 4 B-\mathrm{H} 4 B C \cdots \mathrm{O} 1 A^{\mathrm{ii}}$ & 0.98 & 2.56 & $3.336(4)$ & 136 \\
$\mathrm{C} 1 B-\mathrm{H} 1 B \cdots \mathrm{O} 1 B^{\mathrm{i}}$ & 0.95 & 2.48 & $3.248(4)$ & 138 \\
$\mathrm{C} 2 B-\mathrm{H} 2 B \cdots \mathrm{O} 2 B^{i i \mathrm{ii}}$ & 0.95 & 2.41 & $3.298(4)$ & 155 \\
$\mathrm{C} 4 B-\mathrm{H} 4 B A \cdots \mathrm{O} 1 B^{\mathrm{i}}$ & 0.98 & 2.50 & $3.345(4)$ & 144
\end{tabular}

Symmetry codes: (i) $-x+1, y+1 / 2,-z+2$; (ii) $-x+1, y+1 / 2,-z+1$; (iii) $-x+1, y-1 / 2,-z+2$. 